\title{
Prevalence of cardiac involvement in home-based recovered coronavirus disease 2019 (COVID-19) patients: a retrospective observational study
}

\author{
Özge Çakmak Karaaslan ${ }^{1}$ (1) - Murat Oğuz Özilhan ${ }^{1}$ • Orhan Maden ${ }^{1}$. Omaç Tüfekçioğlu ${ }^{1}$
}

Received: 1 October 2021 / Accepted: 21 October 2021 / Published online: 29 October 2021

(c) The Author(s), under exclusive licence to Royal Academy of Medicine in Ireland 2021

\begin{abstract}
Objective Coronavirus disease 2019 (COVID-19) has a risk of cardiac arrhythmia, acute coronary syndrome, heart failure and myocarditis, and the prognosis of COVID-19 has been associated with cardiovascular symptoms. However, there has not been enough information about cardiovascular involvement in patients who had recovered home-based mild symptoms of COVID-19 infection. Therefore, this study evaluates the prevalence of cardiac involvement and associated factors in home-based recovered COVID-19 patients.

Subject and methods.

A total of 64 participants who applied to cardiology outpatient clinics with cardiac symptoms after recovering from COVID infection were recorded between April and December 2020. The patients were divided into two according to cardiovascular involvement in the cardiovascular magnetic resonance (CMR) imaging results.

Results No significant difference between the two groups regarding age and co-morbidities. Patients with cardiac involvement had higher C-reactive protein compared to without cardiac involvement patients. A total of 46 patients who recently recovered from COVID-19 had abnormal CMR findings such as myocardial late gadolinium enhancement or pericardial enhancement. In addition, the left ventricular ejection fraction and stroke volume were significantly lower in the cardiac involvement patients.

Conclusion We demonstrate cardiac involvement in 46 patients (71\%) with recent COVID-19, independent of pre-existing conditions. This indicates that there may be widespread cardiac involvement without high troponin values or severe clinical symptoms.
\end{abstract}

Keywords Cardiac involvement $\cdot$ Cardiac magnetic resonance $\cdot$ COVID-19 $\cdot$ Myocardial damage

\section{Introduction}

Coronavirus disease 2019 (COVID-19) was first identified in patients complaining of acute respiratory symptoms in Wuhan, China, in December 2019 [1]. It has been reported that severe acute respiratory syndrome coronavirus 2

Özge Çakmak Karaaslan

ozgecakmak2323@gmail.com

Murat Oğuz Özilhan

murat_ozilhan@hotmail.com

Orhan Maden

madenorhan@hotmail.com

1 Department of Cardiology, Cardiology Clinic, Ankara City Hospital, Ankara, Turkey
(SARS-CoV-2) can affect the cardiovascular system directly or indirectly [2]. Although mortality has mainly been due to severe acute respiratory syndrome and multi-organ dysfunction in COVID-19, cardiovascular complications have been demonstrated as the cause of death in some COVID19 patients [3, 4]. COVID-19 disease has a risk of cardiac arrhythmia, acute coronary syndrome, heart failure complications and fulminant myocarditis, and the prognosis of COVID-19 disease has been associated with cardiovascular symptoms [2,5].

It is known that approximately $20 \%$ of all cases remain asymptomatic throughout the disease [6]. The long-term cardiac effect has been reported in patients who recently recovered from COVID-19, regardless of the severity and overall course of the acute disease [7]. In a recent study, $78 \%$ of patients who recovered from COVID-19 infection, 
regardless of cardiac symptoms, had cardiovascular involvement as detected by cardiovascular magnetic resonance (CMR) [7].

There has not been enough information about cardiovascular involvement in patients who have had a COVID-19 infection; those with were not hospitalised or had no symptoms or only mild symptoms. Therefore, the diagnosis of cardiac involvement is critical for the close follow-up, treatment, and awareness of high-risk patients. Therefore, we aimed to evaluate the prevalence of cardiac involvement and associated factors in home-based recovered COVID-19 patients.

\section{Material and methods}

\section{Study design and participants}

This was a retrospective observational study of 64 patients diagnosed with SARS-CoV-2 by the reverse transcription polymerase chain reaction (PCR) on a swab test of the upper respiratory tract. Participants who applied to cardiology outpatient clinics with cardiac symptoms after recovering from COVID infection were recorded between April and December 2020. The patients were divided into two according to the presence of cardiovascular involvement in the CMR imaging results. Cardiac involvement was defined as the presence of myocardial or pericardial late gadolinium enhancement (LGE) or pericardial effusion. All participants were accepted at least two weeks after the original diagnosis when respiratory symptoms resolved and the isolation period ended. In this analysis, patients who had performed CMR and hospitalised for active cardiac symptoms or COVID19 were not included. Exclusion criteria were the presence and history of heart failure with reduced ejection fraction $(\mathrm{EF}<50 \%)$, structural heart disease, moderate or severe valvular heart disease, known as pulmonary arterial hypertension, and individuals with systemic and metabolic disorders that could adversely affect the cardiac functions and particular contraindications for contrast-enhanced magnetic resonance. The study protocol was approved by the institutional ethics committee of our hospital and the Ministry of Health. All procedures were conducted consistently with the Declaration of Helsinki. All the patients provided written informed consent.

The baseline clinical and demographic characteristics of the patients were gathered from the hospital's medical database. All the participants underwent venous blood sampling after admission to cardiology outpatient clinics. The local laboratory cut-off value for high-sensitivity troponin-T (hsTnT) was accepted above the 99th percentile (45 ng/L) counted as a significant increase. Left ventricular ejection fraction (LVEF) was calculated using the modified Simpson method [8].

\section{Cardiac magnetic resonance protocol}

CMR examinations were performed on a 1.5 Tesla system (Signa Explorer, General Electric, Milwaukee, USA). A 16-channel body coil was used for data acquisitions. Cine balanced steady-state free precession (b-SSFP) images were obtained from cardiac base-to-apex as short-axis stack images with retrospective electrocardiogram triggering and breath holding. The image analysis was made on a remote workstation (Advantage Windows, version 4.3, and version 4.4; GE Medical Systems) with cardiac analysis software (ReportCard 2.0; GE Medical Systems). In each patient, enddiastolic volume (EDV), end-systolic volume (ESV), stroke volume and LVEF were calculated, divided by body surface area to obtain indexed right ventricle volumes. LGE imaging was performed approximately $10 \mathrm{~min}$ after administration of body weight-appropriate gadobutrol. To evaluate the LGE, all short-axis slices from basis to apex were assessed for zones of normal myocardium. Scar distribution types were then assessed in accordance with the transmural, focal and diffuse involvement. All images were analysed by one cardiologist. The study was accepted as positive for myocarditis, considering the Lake Louise criteria.

\section{Statistical analysis}

All the data were analysed using the SPSS 22.0 Statistical Package Program for Windows (SPSS; IBM, Armonk, New York, USA). A Kolmogorov-Smirnov test was utilised for the assessment of the normality of distribution. Continuous variables were presented as mean \pm standard deviation and median with interquartile ranges and categorical variables as the number of patients and percentages. A comparison between groups was made with a Student's $t$-test for normally distributed variables and a Mann-Whitney $U$ test for variables without normal distribution. Finally, categorical data from both groups were compared using the $\chi^{2}$ or Fisher's exact test. All tests were 2-tailed, and $p$ values less than $<0.05$ were considered statistically significant.

\section{Results}

A total of 64 patients who recently recovered from COVID-19 infection constituted the study population. The mean (SD) age was 41 (14) years, and the majority of the study group (64\%) were male. Baseline characteristics and blood test results are shown in Table 1. No significant difference between the two groups regarding age and co-morbidities such as hypertension, diabetes, hypercholesterolemia, known coronary artery 
Table 1 Baseline characteristics and blood test results

\begin{tabular}{lllll}
\hline & All $(n: 64)$ & $\begin{array}{l}\text { Cardiac involvement } \\
(-)(n: 18)\end{array}$ & $\begin{array}{l}\text { Cardiac involvement }(+) \\
(n: 46)\end{array}$ & $p$-value \\
\hline Age & $41.4 \pm 14.63$ & $43.33 \pm 16.05$ & $40.62 \pm 14.14$ & .511 \\
Male, $n(\%)$ & $41(64.1)$ & $11(61.1)$ & $30(65.2)$ & .758 \\
Hypertension, $n(\%)$ & $9(14.1)$ & $2(11.1)$ & $7(15.2)$ & .671 \\
Diabetes, $n(\%)$ & $6(9.4)$ & $1(5.6)$ & $5(10.9)$ & .512 \\
Hypercholesterolemia, $n(\%)$ & $5(7.8)$ & $1(5.6)$ & $4(8.7)$ & .674 \\
Known CAD, $n(\%)$ & $6(9.4)$ & $1(5.6)$ & $5(10.9)$ & .512 \\
Smoking, $n(\%)$ & $10(15.6)$ & $2(11.1)$ & $8(17.4)$ & .534 \\
COPD or asthma, $n(\%)$ & $6(9.4)$ & $1(5.6)$ & $5(10.9)$ & .512 \\
Systolic blood pressure, mmHg & $120(100-160)$ & $120(110-160)$ & $120(100-140)$ & .855 \\
Diastolic blood pressure, mmHg & $70(60-100)$ & $80(60-100)$ & $70(60-90)$ & .107 \\
Heart rate, beats per min & $78(56-118)$ & $78,5(65-87)$ & $78(56-118)$ & .654 \\
CRP, mg/dL & $5(1-211)$ & $2.5(1-17)$ & $5(1-211)$ & .048 \\
hsTnT, ng/L & $2.5(2-254)$ & $2.5(2.5-73)$ & $2.5(2-254)$ & .521 \\
Significant elevated hsTnT & $9(14.1)$ & $1(5.6)$ & $8(17.4)$ & .221 \\
$\quad(\geq 45$ ng/L), n (\%) & & & $35(35-4681)$ & .166 \\
NT-pro BNP, ng/L & $35(35-4681)$ & $35(35-315)$ & & \\
\hline
\end{tabular}

Continuous data are presented as mean \pm standard deviation, or median (interquartile range)

COVID-19 coronavirus disease 2019, CAD coronary artery disease, COPD chronic obstructive pulmonary disease, $C R P$ C-reactive protein, $h s T n T$ high-sensitivity troponin T, NT-pro BNP N-terminal pro-b-type natriuretic peptide disease, smoking and chronic obstructive pulmonary disease or asthma was noted. There were no differences in systolic, diastolic blood pressure values and heart rate between the groups. Patients with cardiac involvement had higher C-reactive protein (CRP) compared with no-cardiac involvement group $(p=.048)$. There were no significant differences regarding hsTnT and $\mathrm{N}$ terminal pro-b-type natriuretic peptide between groups. Nine patients $(14.1 \%)$ had significantly elevated hsTnT ( $>45 \mathrm{pg} / \mathrm{mL})$.

The most common complaint was chest pain $(n=43)$. In this study, none of the patients received mechanical ventilation or non-invasive ventilation with positive airway pressure. There is no significant difference between groups about COVID-19 treatment protocol including favipiravir, hydroxychloroquine, glucocorticoid and azithromycin (Table 2).

A total of 46 patients who recently recovered from COVID- 19 diseases had abnormal CMR findings such as myocardial LGE or pericardial enhancement (Table 3). The LVEF values $(p=0.008)$ and stroke volume $(p=0.029)$ were significantly lower in the cardiac involvement group. There were no differences in EDV, ESV and tricuspid annular plane systolic excursion values among groups. In particular, in these patients, the late gadolinium enhancement was commonly located in the left ventricle's inferior (58\%) and lateral $(39.1 \%)$ walls. Pericardial effusion was detected in 7 patients, but none was greater $\geq 10 \mathrm{~mm}$. The median time interval between COVID-19 diagnosis and CMR was 71
Table 2 Presenting symptoms and treatment of management COVID-19

\begin{tabular}{lllll}
\hline & All $(n: 64)$ & $\begin{array}{l}\text { Cardiac involvement } \\
(-)(n: 18)\end{array}$ & $\begin{array}{l}\text { Cardiac involvement } \\
(+) \\
(n: 46)\end{array}$ & $p$-value \\
\hline $\begin{array}{l}\text { Presenting symptoms } \\
\text { Chest pain, } n(\%)\end{array}$ & $43(67.1)$ & $14(22.2)$ & $29(37.0)$ & .259 \\
$\begin{array}{l}\text { Shortness of breath/dyspnea, } n(\%) \\
\text { Management of COViD-19 }\end{array}$ & $24(37.5)$ & $6(33.3)$ & $18(39.1)$ & .667 \\
Favipiravir, $n(\%)$ & $51(79.6)$ & $17(94.4)$ & $34(73.9)$ & .089 \\
Hydroxychloroquine, $n(\%)$ & $48(75)$ & $15(83.3)$ & $33(71.7)$ & .336 \\
Glucocorticoids, $n(\%)$ & $2(3.125)$ & $1(5.6)$ & $1(2.2)$ & .485 \\
Azithromycin, $n(\%)$ & $10(15.6)$ & $2(11.1)$ & $8(17.4)$ & .534 \\
\hline
\end{tabular}

COVID-19 coronavirus disease 2019 
Table 3 Cardiac magnetic resonance imaging and echocardiogram findings
Cardiac involvement Cardiac involvement $(+) \quad p$-value $(-)(n: 18) \quad(n: 46)$

\begin{tabular}{|c|c|c|c|}
\hline \multicolumn{4}{|l|}{ CMR findings } \\
\hline LVEF & $67(58-76)$ & $62(30-72)$ & .008 \\
\hline Stroke volume & $73.56 \pm 18.87$ & $64.67 \pm 17.08$ & .029 \\
\hline $\mathrm{EDV}(\mathrm{mL})$ & $112.06 \pm 26.47$ & $107.72 \pm 28.40$ & .861 \\
\hline $\mathrm{ESV}(\mathrm{mL})$ & $33.5(24-62)$ & $41(17-107)$ & .059 \\
\hline TAPSE (mm) & $22.5(20-26)$ & $23(15-27)$ & .539 \\
\hline LGE, $n(\%)$ & - & 46 & \\
\hline \multicolumn{4}{|l|}{ LGE segment } \\
\hline *Anterior & & $7(15.2)$ & \\
\hline *Lateral & & $18(39.1)$ & \\
\hline *Posterior & & $15(32.6)$ & \\
\hline *Inferior & & $27(58.6)$ & \\
\hline *Septum & & $6(13)$ & \\
\hline *Pericardial & & $11(23.9)$ & \\
\hline Pericardial effusion & & $7(15.2)$ & \\
\hline $\begin{array}{l}\text { The median time interval between } \\
\text { COVID-19 diagnosis and CMR }\end{array}$ & $71(17-209)$ & $66(17-150)$ & $69(20-209)$ \\
\hline \multicolumn{4}{|l|}{ Echocardiogram findings } \\
\hline LVEF & $62(60-65)$ & $60(35-65)$ & .003 \\
\hline
\end{tabular}

Continuous data are presented as mean \pm standard deviation, or median (interquartile range)

COVID-19 coronavirus disease 2019, LVEF left ventricular ejection fraction, $E D V$ end-diastolic volume, $E S V$ end-systolic volume, TAPSE tricuspid annular plane systolic excursion, $L G E$ late gadolinium enhancement, $C M R$ cardiac magnetic resonance
(17-209) days. In the cardiac involvement, the patients were observed to have reduced LVEF as an echocardiographic finding $(p=.003)$.

\section{Discussion}

A total of 64 patients of which were mildly symptomatic and recovered at home from COVID-19 were included in this study. A total of 46 patients $(71 \%)$ were detected to have cardiac involvement, according to CMR imaging irrespective of pre-existing conditions, presenting cardiac symptoms or the severity and overall course of the COVID19 presentation. The patients with cardiac involvement had mildly reduced LVEF and left ventricular stroke volume compared to those without cardiac involvement. Although LVEF as echocardiogram finding was within normal values in both groups, the patients with cardiac involvement were observed to have reduced LVEF with unclear clinical significance.

It has been known that myocardial damage occurs in 20-30\% of patients admitted to hospital for COVID-19 [9]. In COVID-19, numerous mechanisms affect the cardiovascular system and caused cardiac hemodynamic disorders $[10,11]$. Likely mechanisms by which COVID-19 leads to cardiovascular damage include direct myocardial injury resulting from the inflammatory cascade or cytokine release, microvascular damage and supply/demand imbalance [12]. Increased sympathetic stimulation has increased myocardial oxygen consumption, and hypoxia has decreased myocardial oxygen delivery [11]. A small number of post-mortem cardiac biopsy examinations have been shown several interstitial mononuclear inflammatory cell infiltrations in myocardial tissue, macroscopically cardiomegaly, enlargement of right heart chambers, microscopically in myocardial tissue distributed, small areas of myocyte necrosis, lymphocyte infiltration and myocardial oedema [13, 14].

CMR provides detailed tissue characterisation, including necrosis, scar, hyperaemia, contractile dysfunction, diffuse fibrosis and oedema [15]. The LGE in the zones with increased contrast agent uptake indicates regional damage due to myocardial inflammation. Non-ischemic states of myocardial LGE are mainly observed in patients with myocarditis and have been strongly associated with poor outcomes [16]. The presence of pericardial effusion, fibrosis or oedema may be found secondary to ongoing pericarditis [7]. It has been known that increased native T1 and T2 measures indicated myocardial fibrosis and oedema [17]. A retrospective multi-centre cohort study of approximately 3000 patients reported that myocardial injury was often mild 
and common among patients hospitalised with COVID-19 and was associated with a low-level elevation in troponin concentration [12].

Cardiac involvement among symptomatic, hospitalised patients with COVID-19 is known to occur in over half, and LGE in nearly one-third of all patients [18], but its prevalence is unclear in home-based recovered COVID-19 patients. Puntmann et al. demonstrated that in a total of 100 patients of COVID 19 assessed by CMR, 78\% of the patients had an abnormal finding in CMR and one-third required hospitalisation, while the rest recovered at home [7].

This study provides important insights into the prevalence of cardiac involvement in patients with home-based recovery from COVID-19. Our results demonstrated that cardiac involvement was prevalent in patients with home-based recovery from COVID-19. Previously, it has been reported that sudden cardiac death likely occurred in non-hospitalised patients with mild symptoms who were in home quarantine [19]. These sudden deaths have demonstrated the need for data about the cardiac involvement of COVID-19. In our study, we observed an increased contrast agent uptake despite normal systolic parameters measured by CMR and conventional echocardiography. It has been known that LGE, which reflects limited myocyte necrosis, has been demonstrated less frequently in these patients [20]. Even so, $70 \%$ of patients in the study cohort were observed to have LGE. This result was concordant with early case reports in hospitalised patients showing a frequent presence of LGE [21].

In previous case reports, patients with COVID-19-associated myocarditis were detected to have diffused myocardial wall oedema and reduced LVEF in the CMR [21, 22]. On the contrary, the CMR results of our patients had demonstrated mainly focal myocardial oedema and mostly normal left ventricular function and volumes. This indicates that there may be widespread myocardial involvement without high hsTnT values or severe clinical symptoms.

This study was significant because it has provided knowledge about the prevalence of cardiac involvement in mildly symptomatic COVID-19 patients. Our results indicate considerable awareness of the prevalence of cardiac involvement irrespective of troponin values. Increased troponin levels in COVID-19 disease may reflect the severity of the disease rather than cardiac involvement. We consider that most patients had cardiac involvement, regardless of the severity and overall course of the acute disease.

Our findings support that patients with mild symptomatic COVID-19 infection may have subclinical cardiac involvement.

Our study has limitations. Myocardial T1 and T2 mapping could not be performed, so using other imaging protocols or sequence parameters may provide different results.

\section{Conclusion}

This study provides important insights into the prevalence of cardiac involvement in patients with home-based recovery from COVID-19. Our results indicate considerable awareness of the prevalence of cardiac involvement. Furthermore, these insights show the need for ongoing investigation of the long-term cardiovascular outcomes of COVID-19.

Author contribution All the authors contributed to the study conception and design. ÖÇK: project development, data collection, statistical analysis and manuscript writing. MOO: project development and manuscript writing. OM, OT: supervision and review. All the authors read and approved the final manuscript.

\section{Declarations}

Ethical approval This study was approved by the Health Sciences University Ankara City Hospital Ethics Committee and the Ministry of Health, dated 3.02.2021, No: E1-21-1488.

Consent to participate Informed consent was obtained from all individual participants included in the study.

Conflict of interest The authors declare no competing interests.

\section{References}

1. Zhu N, Zhang D, Wang W et al (2020) A novel coronavirus from patients with pneumonia in China. N Engl J Med 382(8):727-733. https://doi.org/10.1056/NEJMoa2001017

2. Kang Y, Chen T, Mui D et al (2020) Cardiovascular manifestations and treatment considerations in COVID-19. Heart (British Cardiac Society) 106(15):1132-1141. https://doi.org/10.1136/ heartjnl-2020-317056

3. Mahase E (2020) Covid-19: death rate is $0.66 \%$ and increases with age, study estimates. BMJ (Clinical research ed) 369:m1327. https:// doi.org/10.1136/bmj.m1327

4. Ruan Q, Yang K, Wang W et al (2020) Clinical predictors of mortality due to COVID-19 based on an analysis of data of 150 patients from Wuhan. China Intensive care medicine 46(5):846848. https://doi.org/10.1007/s00134-020-05991-x

5. Guo T, Fan Y, Chen M et al (2020) Cardiovascular implications of fatal outcomes of patients with coronavirus disease 2019 (COVID19). JAMA cardiology 5(7):811-818. https://doi.org/10.1001/ jamacardio.2020.1017

6. Buitrago-Garcia D, Egli-Gany D, Counotte MJ et al (2020) Occurrence and transmission potential of asymptomatic and presymptomatic SARS-CoV-2 infections: a living systematic review and meta-analysis. PLoS Med 17(9):e1003346. https://doi.org/10. 1371/journal.pmed.1003346

7. Puntmann VO, Carerj ML, Wieters I et al (2020) Outcomes of cardiovascular magnetic resonance imaging in patients recently recovered from coronavirus disease 2019 (COVID-19). JAMA cardiology 5(11):1265-1273. https://doi.org/10.1001/jamacardio.2020.3557

8. Schiller NB, Shah PM, Crawford M et al (1989) Recommendations for quantitation of the left ventricle by two-dimensional 
echocardiography. American Society of Echocardiography Committee on Standards, Subcommittee on Quantitation of TwoDimensional Echocardiograms. Journal of the American Society of Echocardiography : official publication of the American Society of Echocardiography 2 (5):358-367. https://doi.org/10.1016/ s0894-7317(89)80014-8

9. Shi S, Qin M, Shen B et al (2020) Association of cardiac injury with mortality in hospitalized patients With COVID-19 in Wuhan. China JAMA cardiology 5(7):802-810. https://doi.org/10.1001/ jamacardio.2020.0950

10. Madjid M, Safavi-Naeini P, Solomon SD, Vardeny O (2020) Potential effects of coronaviruses on the cardiovascular system: a review. JAMA cardiology 5(7):831-840. https://doi.org/10.1001/ jamacardio.2020.1286

11. Xiong TY, Redwood S, Prendergast B, Chen M (2020) Coronaviruses and the cardiovascular system: acute and long-term implications. Eur Heart J 41(19):1798-1800. https://doi.org/10.1093/ eurheartj/ehaa231

12. Lala A, Johnson KW, Russak AJ et al (2020) Prevalence and impact of myocardial injury in patients hospitalized with COVID19 Infection. medRxiv : the preprint server for health sciences. https://doi.org/10.1101/2020.04.20.20072702

13. Xu Z, Shi L, Wang Y et al (2020) Pathological findings of COVID-19 associated with acute respiratory distress syndrome. Lancet Respir Med 8(4):420-422. https://doi.org/10.1016/s22132600(20)30076-X

14. Fox SE, Akmatbekov A, Harbert JL et al (2020) Pulmonary and cardiac pathology in African American patients with COVID-19: an autopsy series from New Orleans. Lancet Respir Med 8(7):681-686. https://doi.org/10.1016/s2213-2600(20)30243-5

15. Bhatia S, Anstine C, Jaffe AS et al (2019) Cardiac magnetic resonance in patients with elevated troponin and normal coronary angiography. Heart (British Cardiac Society) 105(16):1231-1236. https://doi.org/10.1136/heartjnl-2018-314631
16. Gulati A, Jabbour A, Ismail TF et al (2013) Association of fibrosis with mortality and sudden cardiac death in patients with nonischemic dilated cardiomyopathy. JAMA 309(9):896-908. https:// doi.org/10.1001/jama.2013.1363

17. Puntmann VO, Valbuena S, Hinojar R et al (2018) Society for Cardiovascular Magnetic Resonance (SCMR) expert consensus for CMR imaging endpoints in clinical research: part I - analytical validation and clinical qualification. Journal of cardiovascular magnetic resonance : official journal of the Society for Cardiovascular Magnetic Resonance 20(1):67. https://doi.org/10.1186/s12968-018-0484-5

18. Huang L, Zhao P, Tang D et al (2020) Cardiac involvement in patients recovered from COVID-2019 identified using magnetic resonance imaging. JACC Cardiovasc Imaging 13(11):2330-2339. https://doi.org/10.1016/j.jcmg.2020.05.004

19. Kochi AN, Tagliari AP, Forleo GB et al (2020) Cardiac and arrhythmic complications in patients with COVID-19. J Cardiovasc Electrophysiol 31(5):1003-1008. https://doi.org/10.1111/jce. 14479

20. Esposito A, Palmisano A, Natale L et al (2020) Cardiac magnetic resonance characterization of myocarditis-like acute cardiac syndrome in COVID-19. JACC Cardiovasc Imaging 13(11):24622465. https://doi.org/10.1016/j.jcmg.2020.06.003

21. Inciardi RM, Lupi L, Zaccone G et al (2020) Cardiac Involvement in a patient with coronavirus disease 2019 (COVID-19). JAMA cardiology 5(7):819-824. https://doi.org/10.1001/jamacardio. 2020.1096

22. Kim IC, Kim JY, Kim HA, Han S (2020) COVID-19-related myocarditis in a 21-year-old female patient. Eur Heart J 41(19):1859. https://doi.org/10.1093/eurheartj/ehaa288

Publisher's Note Springer Nature remains neutral with regard to jurisdictional claims in published maps and institutional affiliations. 